\title{
Optimizing multidisciplinary scaled tests in terrestrial atmosphere for extraterrestrial unmanned aerial vehicle missions
}

\author{
Ana I Moreno, Artur Jarzabek, Miguel A González and \\ José M Perales
}

\begin{abstract}
Unmanned aerial vehicles are a valid tool for exploring celestial objects that have atmosphere. The design process of these vehicles includes numerical analysis phase, which is then followed by an experimental flight test campaign in order to validate and refine the design. In terrestrial conditions, it is impossible to use the full size prototype and recreate all the conditions of extraterrestrial flight. The only option is to use a scaled model maintaining certain similarity parameters to the prototype. This would, however, not reproduce the global multidisciplinary behavior in terrestrial conditions. Alternatively, relaxing similarity constraints enables reproducing the global multidisciplinary behavior but introduces known and measurable similarity differences. The objective of this study is to provide a methodology for designing a relaxed similarity scaled model for terrestrial multidisciplinary tests for any extraterrestrial unmanned aerial vehicle. The methodology is then applied to a case scenario in order to verify its validity.
\end{abstract}

\section{Introduction}

Scientific interest in exploring celestial bodies in the Solar System using unmanned vehicles has been constantly growing since the launch of the first satellite in 1957. All of the vehicles sent to investigate the Solar System objects were either satellites, probes, or rovers with limited movement and control capability. A yet untested possibility of exploration is to use controlled planetary flight vehicles (PFV). A crucial phase of the design of such vehicles is flight testing. Its purpose is to both confirm the validity of the original design, usually based on numerical analysis, and to introduce necessary changes based on the obtained data. These data may also serve for implementing semi-empirical models in the theoretical design phase. However, it is very difficult to reproduce on Earth the same mission conditions and in most of the cases scaled models are essential because, as Savu demonstrated for Mars, full size prototypes optimized for Earth are not capable of flying in extraterrestrial atmospheres.

$\mathrm{Liu}^{2}$ showed that using power laws parameters such as geometry, velocity, and power required for flight can be correlated against each other for both terrestrial flying vehicles and animals (birds, insects, etc.). This idea has been further expanded to planetary exploration by Lorenz. ${ }^{3}$ This proves that there exists a possibility for a vehicle, which is to fly in extraterrestrial atmosphere, to be adequately scaled to reproduce the same behavior in terrestrial conditions. In fact scaling, based on nondimensional parameters of dynamic similarity obtained using the Buckingham Pi Rule, ${ }^{4}$ is a commonly used method during the design process of extraterrestrial vehicles as Savu details. ${ }^{1}$ The main reason is that it allows to predict vehicle's behavior at its destination interpreting results obtained from experimental terrestrial methods using scaled models that maintain the mentioned dynamic similarity. Scaling has been applied in predicting the terminal descent dynamics of the Mars Exploration Rover Mission, by Mitcheltree, ${ }^{5}$ which cannot be tested using a full size original prototype. 
In the case when it is possible, it has to be done at very high altitudes and at supersonic speeds or in facilities such as the NASA's Planetary Aeolian Laboratory, ${ }^{6}$ that faithfully reproduce given atmosphere condition. The ARES Mars Scout Mission ${ }^{7}$ is one example of that when its design was based on data from a very sophisticated test plan that included high altitudes flight tests and diverse tests in the Aurora's Ultra High Altitude Test Facility. However, high level of test sophistication usually proves to be a technical challenge which leads directly to high costs, which sometimes exceed the project's budget. Consequently, testing the descent dynamics and parachute qualification relies heavily on scaled wind tunnel models or low altitude tests before applying extrapolation algorithms based on previous high altitude flight tests. ${ }^{8}$ Lastly, there are a few authors, such as Liu, ${ }^{9}$ who use model scaling power laws to provide a methodology of scaling unmanned aerial vehicle (UAV) from terrestrial to Martian conditions.

Summarizing, scaling is a feasible method of testing extraterrestrial designs in terrestrial conditions. Moreover, diverse scaling strategies have been already implemented. Nevertheless, regardless of the method used, the scaled model exactly reproduces the prototype's behavior because it applies similarity to the discipline parameters which define the system as determined. This means leaving other disciplines and their parameters uncontrolled. In order to mitigate the undesired inconsistencies in other disciplines reproduction, the restrictions imposed by similarity methodology need to be relaxed. Such a scaling procedure would allow taking into account all parameters involved in the system to be scaled, solving the problem by introducing redundancy and at the same time increasing the feasibility of model testing. This could be done by appropriately defining an optimization problem. This would come at a cost of reproducing the prototype behavior with known and measurable differences. Additionally, those differences can be minimized by identifying critical disciplines and optimizing the relaxation factors based on parameter sensitivity. For the purpose of differentiating both methodologies, from now on the similarity scaling will be referred to as "strict similarity" and relaxed scaling "relaxed similarity".

Unlike previous works, ${ }^{1,5,9}$ this study aims to define a multidisciplinary scaling procedure. Since strict scaling is a trivial problem, this article is focused on optimizing relaxed scaling law giving a methodology background and applying it to a case study based on the Titan exploration UAV project AVIATR.$^{10,11}$ The optimized disciplines include aerodynamics, structure and propeller performance, but can be further expanded if needed.

\section{Extraterrestrial flight}

Since intrusive exploration is limited by relatively large distances between objects in space, the domain of extraterrestrial missions have been limited to the Solar System. This domain can be further narrowed to Mars, Titan, and Venus which, as described below, are most suitable for extraterrestrial flight.

\section{UAV projects}

Aerial vehicles present certain advantages in comparison to surface vehicles which were demonstrated by successful missions with balloons and probes. ${ }^{12,13}$ A new generation of balloon explorers (Directed Aerial Robot Explorers, DAREs) are being developed by Pankine ${ }^{14}$ and has been recently proposed by Lorenz for Titan missions. ${ }^{15}$ The advantages of balloons is their low technological cost and low energy consumption but their mobility is limited in comparison to fixed-wing aircrafts or rotorcrafts. Several authors share this opinion, in particular for Titan exploration. ${ }^{16} 19$ They recognize their limitations proposing aircraft or rotorcraft configurations as feasible stand-alone platforms. Autonomous vehicles are able to freely explore, which is an improvement in extraterrestrial scientific research techniques, as Rhew states in his Mars PFVs study ${ }^{20}$ and Young outlines in relation to VTOL vehicles. ${ }^{21}$ Miralles $^{22}$ also analyzed possible scenarios for planetary gliders.

In terms of Martian exploration, the Aerial Regional-scale Environmental Survey of Mars (ARES) vehicle is proposed for the Mars Scout mission. ${ }^{23}$ At present, the project has reached wind tunnel tests and high altitude flight tests stage. ${ }^{7,20}$ The project implementation is due in $2018 .^{24}$ Regarding Titan, numerous projects of atmospheric flight vehicles have been proposed $d^{3,10,11,25} 27$ but the most developed one is the AVIATR (Aerial Vehicle of In-Situ and Airborne Titan Reconnaissance) project developed by Barnes with the objective of studying Titan's geography and atmosphere. It is the most representative design of Titan exploration vehicles as it has reached the furthest stage of development. Venus has been already successfully explored by two UAVs which were Vega Mission 1 and 2 balloons sent in 1984 by the Russian Space Agency. ${ }^{12}$ Recently the European Venus Explorer mission, ${ }^{28}$ which is a single balloon probe, has been proposed by ESA to investigate the planet's climate, formation and evolution. Aerial explorers are in the stage of conceptual planning. One example is NASA's project of a solar-powered aircraft developed by Landis. ${ }^{29}$

\section{Atmosphere modeling}

Since atmospheric properties and gravity directly influence flight dynamics, the first step to define the scaling law is extraterrestrial atmosphere and gravity modeling. Atmosphere can be considered as a uniformly distributed ideal gas and therefore its properties can be parametrized with flight altitude obtaining a model analogous to the widely used Earth's 
Standard Atmosphere. Gravity acceleration can be assumed constant within the flight envelope. Correlation of extraterrestrial and terrestrial flight conditions is what mainly defines the feasibility of using a strict similarity scaled model.

Mars has been explored from the early $1970 \mathrm{~s}^{30}$ with the intention of characterizing its atmosphere. In 1996, the Mars Global Surveyor mission was launched to do a global mapping of the planet which included the atmosphere. ${ }^{31}$ Mars has a very thin atmosphere mainly composed of carbon dioxide and an accurate parametric model is available in the NASA archives. ${ }^{32}$ Titan atmosphere is composed mainly of nitrogen which can be assumed as pure nitrogen gas in first approximation. ${ }^{16,33}$ The main atmospheric properties are obtained from the tabulated Lellouch-Hunten Titan atmosphere. ${ }^{34}$ Venus has a highly dense atmosphere consisting of $93-97 \%$ $\mathrm{CO}_{2}$ and its main atmospheric properties as density, pressure and temperature are available as tabulated data from missions measurements Venera 5 and $6 .{ }^{35}$ For all the objects the speed of sound and dynamic viscosity can be derived using the ideal gas law and the Sutherland's relationship, ${ }^{36}$ respectively.

A combination of terrestrial and extraterrestrial altitudes generates a surface map of property ratios which are hereinafter referred to as $N_{i}$, where the index $i$ can take values of the property's conventional symbol, i.e. $g, \rho, v$, and $a$ stand for gravitational acceleration, density, kinematic viscosity and speed of sound respectively. For example $N_{\rho}=\rho_{\text {earth }} / \rho_{\text {object }}$ is the ratio between terrestrial density and the corresponding planetary one as a function of both altitudes.

Once the mission altitude in the extraterrestrial planet is defined, the surface map of property ratios is intersected by the extraterrestrial altitude plane. This intersection is a 2D curve which is then used to identify the altitude on Earth for a terrestrial flight which strictly reproduces mission conditions or is the most optimal. Atmospheric properties and gravity ratios, given in Table 1, are very different in each case making the design process of the flight vehicle unique for each object, justifying the need to validate the final design with flight tests of models in Earth. Additionally, $N_{i}$ ratios deviating excessively from unity make full size prototype testing impossible in terrestrial conditions.

\section{Scaling methodology}

Same as for any dynamic system, using a scaled aircraft in cruise flight requires ensuring geometrical, kinematic and dynamic similarity. Geometric scaling means that the model is uniformly scaled maintaining all the geometric length ratios and angles of the full size prototype. Kinematic similarity ensures that the streamlines patterns are maintained. Dynamic similarity means that forces and moments are uniformly scaled. Maintaining those criteria, which are governed
Table I. Gravity ratios, $N_{g}$.

\begin{tabular}{ll}
\hline Planets & $N_{g}$ \\
\hline Titan & 7.26 \\
Venus & 1.11 \\
Mars & 2.64 \\
\hline
\end{tabular}

by certain nondimensional parameters, ensures that the dynamic behavior of the scaled model can be mapped onto the full size prototype. The most common application of dynamic similarity is wind tunnel testing, where geometrically scaled models are analyzed keeping flow angles, Reynolds and/or Mach numbers identical in order that the obtained aerodynamics coefficients are equal.

The use of scaled models is very common in experimental tests studying each of the disciplines of an airplane separately. Flight tests are used to investigate all disciplines simultaneously and thus recreating the global behavior. However, it is difficult to simultaneously scale all disciplines.

Exploration missions require low flight speed, which means flying in incompressible flow regime. This is one of the assumptions that need to be considered. This topic is further expanded in "Feasibility of the scaling law" section.

In order to scale aerodynamics, the Buckingham $\mathrm{Pi}$ Theorem ${ }^{37}$ is applied to the resultant aerodynamic force in cruise flight, $F_{a}$, defined in equation (1) which depends on density, reference length, reference speed, dynamic viscosity, gravity and angle of attack, in order of appearance. This allows to obtain main Pi-groups that define the scaling law of aerodynamics.

$$
f\left(F_{a}, \rho, L, U, \mu, g, \alpha\right)=0
$$

Equation (1) can be described using three fundamental physical units (time, length, and mass). Consequently, the seven arguments in equation (1) can be reduced to four Pi-dimensionless groups using density, length, and speed $(\rho, L$, and $U)$ as dimensionalizing variables. The resulting equation is shown as follows

$$
\Psi\left(\frac{F_{a}}{\rho U^{2} L^{2}}, \frac{\mu}{\rho U L}, \frac{L g}{U^{2}}, \alpha\right)=\Psi\left(C_{F_{a}}, \frac{1}{R e_{L}}, \frac{1}{F r}, \alpha\right)=0
$$

Keeping $R e$ and $F r$ identical ensures dynamic similarity. This combined with kinematic similarity, which in cruise conditions is forced maintaining the angle of attack, $\alpha$, ensures that the pressure distribution over the model is also identical.

The dimensional analysis of the propulsion system is performed in an analogous way by non-dimensionalizing thrust, $T$, shown in equation (3), depending on density, propeller diameter, reference speed, 
dynamic viscosity, and rotational speed, in order of appearance.

$$
f(T, \rho, D, U, \mu, n)=0
$$

Taking density, rotational speed, and propeller diameter $(\rho, n$, and $D)$ as dimensionalizing variables, three Pi-dimensionless groups will be obtained shown as follows

$$
\Psi\left(\frac{T}{\rho n^{2} D^{4}}, \frac{U}{n D}, \frac{\mu}{\rho n D^{2}}\right)=\Psi\left(C_{T}, J, \frac{1}{R e_{D}}\right)=0
$$

In this case, the dynamic similarity parameters are $J$ and $\operatorname{Re}_{D}$ and keeping the flow angles (neglected for the propulsion analysis) maintains kinematic similarity.

Unlike aerodynamics and propulsion, structure is a discipline linked to the dimensions of the model and, therefore, it cannot be described by dimensionless parameters which could maintain structural similarity. As a result, structural design is usually defined once other disciplines, such as aerodynamics, are defined. Ensuring dynamic and geometrical similarity forces stress distribution scaling as stress depends on dynamic forces normalized by geometrical area. Consequently, once the scaling law is defined only checking its impact on structure can be analyzed, because including this relation in the global equation system transforms it into an overdetermined system. Wing loading $W / S$ is the most characteristic parameter representing same physical dimensions as the stress distribution and therefore will be used as the main structural similarity parameter.

\section{Strict similarity scaling}

In order to analyze the global similarity, the Buckhingham $\mathrm{Pi}$ Theorem has to be applied to the global system in trimmed flight where no resultant forces are present. This system is represented by equation (5) which results from merging equations (1) and (3), omitting forces and repeating terms

$$
f(\rho, L, U, \mu, g, \alpha, D, n)=0
$$

Applying the Buckhingham Pi method gives the following equation

$$
\begin{gathered}
\Psi\left(\frac{\mu}{\rho U L}, \frac{L g}{U^{2}}, \alpha, \frac{D}{L}, \frac{n D}{U}\right)=0 \\
\Psi\left(\frac{1}{\operatorname{Re}_{L}}, \frac{1}{F r}, \alpha, \frac{D}{L}, \frac{n L}{U}\right)=0
\end{gathered}
$$

In order to strictly scale the aerodynamic and propulsion disciplines the similarity parameters need to remain constant. It can be deduced from equation (2) that $C_{F a}$ is a function of $R e_{L}, F r$, and $\alpha$ and maintaining all those parameter constant is equivalent to maintaining coefficient of lift $C_{L}$ constant as it is proportional to $C_{F a}$. Since $\alpha$ is not a meaningful parameter from the point of view of scaling methodology, $C_{L}$ will be used in order to scale the mass of the aircraft. According to equation (2) the coefficient of lift can be defined as $C_{L}=L /\left(\rho U^{2} S_{r e f}\right)$ and since trimmed flight is considered, lift must be equal to weight. Hence $C_{L}=m g /\left(\rho U^{2} S_{r e f}\right)$. The similarity condition to simultaneously scale aerodynamics and propulsion is given by the following equation

$$
\left\{\begin{array} { c } 
{ \frac { R e _ { L } | _ { \text { model } } } { R e _ { L } | _ { \text { prototype } } } = N _ { R e _ { L } } = 1 } \\
{ \frac { F r | _ { \text { model } } } { F r | _ { \text { prototype } } } = N _ { F r } = 1 } \\
{ \frac { C _ { L } | _ { \text { model } } } { C _ { L } | _ { \text { prototype } } } = N _ { C _ { L } } = 1 } \\
{ \frac { D / L | _ { \text { model } } } { D / L | _ { \text { prototype } } } = 1 } \\
{ \frac { n L / U | _ { \text { model } } } { n L / U | _ { \text { prototype } } } = 1 }
\end{array} \Rightarrow \left\{\begin{array}{c}
\frac{N_{U} N_{L}}{N_{\nu}}=1 \\
\frac{N_{U}^{2}}{N_{g} N_{L}}=1 \\
\frac{N_{M} N_{g}}{N_{o} N_{U}^{2} N_{L}^{2}}=1 \\
\frac{N_{D}}{N_{L}}=1 \\
\frac{N_{n} N_{L}}{N_{U}}=1
\end{array}\right.\right.
$$

From equation (7) it can be clearly seen that the aerodynamic similarity is fulfilled as it includes all the similarity parameters given in equation (2). To check propulsion similarity equation (7) needs to be rearranged. The fourth line shows that $N_{D}=N_{L}$ and hence substituting $N_{L}$ in the last line gives $N_{n} N_{D} / N_{U}$ which is equivalent to $\frac{\left.f\right|_{\text {prototype }}}{\left.f\right|_{\text {model }}}=N_{J}^{-1}=1$. Substituting $N_{U}$ in the first line by $N_{n} N_{L}$ and using the fact that $N_{D}=N_{L}$ gives $N_{n} N_{D}^{2} / N_{\nu}$ which is equivalent to $\frac{\left.R e_{D}\right|_{\text {model }}}{\left.R e_{D}\right|_{\text {prototipe }}}=N_{R e_{D}}=1$. Consequently, equation (7) does fulfill equation (4) as well.

The vertical force equilibrium is imposed by equaling lift to weight and holds for both the prototype and the model. The horizontal equilibrium requires thrust to be equal to drag which is given by equation (8) where drag and thrust are defined as in equation (2) and (4), respectively

$$
C_{D}\left(\operatorname{Re}_{L}, F r, \alpha\right) \rho U^{2} S_{r e f}=C_{T}\left(\operatorname{Re}_{D}, J\right) \rho n^{2} D^{4}
$$

Cancelling $\rho$ from both sides and transforming equation (8) using ratios between the scaled model and the prototype gives the following

$$
N_{C_{D}} N_{U}^{2} N_{L}^{2}=N_{C_{T}} N_{n}^{2} N_{D}^{4}
$$

Since $C_{D}$ and $C_{T}$ are functions of $R e_{L}, F r, \alpha$ and $R e_{D}, J$ respectively, and all those parameters are maintained constant, $N_{C_{D}}=1$ and $N_{C_{T}}=1$. Additionally, from equation (7) $N_{L}=N_{D}$. Hence equation (9) becomes equation (10)

$$
\frac{N_{U}^{2}}{N_{n}^{2} N_{D}^{2}}=N_{J}^{2}=1
$$


The condition for horizontal trim is fulfilled because as, it has been shown, scaling the model according to equation (7) maintains $N_{J}=1$. Consequently, strict similarity scaling ensures trim in the scaled model as well.

As a consequence of incompressibility assumption, Mach number is not an independent quantity of the scaling law system, but defines a bound condition, ensuring both systems work in the same compressibility regime. The rule of thumb is that Mach number cannot exceed 0.3. Equation (11) shows Mach number ratio for the model and for the tip of the propeller which also needs to satisfy that condition.

$$
\begin{aligned}
& \frac{\text { Mach }\left.\right|_{\text {model }}}{\text { Mach }\left.\right|_{\text {prototype }}}=N_{\text {Mach }}<\frac{0.3}{\text { Mach }\left.\right|_{\text {prototype }}} \\
& \frac{\text { Mach }\left._{t i p}\right|_{\text {model }}}{\text { Mach tip }_{\text {prototype }}}=N_{\text {Mach }_{\text {tip }}}<\frac{0.3}{\text { Mach }\left._{\text {tip }}\right|_{\text {prototype }}} \\
& \text { where } \quad \text { Mach }=\frac{U}{a} \text { and } \text { Mach }_{t i p}=\frac{2 \pi n D}{a}
\end{aligned}
$$

Rearranging the equation system (7) defines the strict similarity scaling ratios given in the following equation

$$
\left\{\begin{array}{l}
N_{U}=\left(N_{g} N_{v}\right)^{1 / 3} \\
N_{L}=\frac{N_{v}^{2 / 3}}{N_{g}^{1 / 3}} \\
N_{M}=\frac{N_{\rho} N_{v}^{2}}{N_{g}} \\
N_{D}=\frac{N_{v}^{2 / 3}}{N_{g}^{1 / 3}} \\
N_{n}=\frac{N_{g}^{2 / 3}}{N_{\nu}^{1 / 3}}
\end{array}\right.
$$

All the ratios depend only on atmospheric parameters and gravity values. This means that the scaling law is independent of the prototype design and depends only on terrestrial flight altitude.

Once the strict scaling strategy is defined, the wing loading ratio can be calculated as a function of the independent parameters of the scaling law.

$$
N_{W / S}=\frac{N_{M} N_{g}}{N_{L}^{2}}=N_{\rho}\left(N_{g} N_{\nu}\right)^{2 / 3}
$$

In order to maintain structural similarity, equation (13) needs to equal one, but for the case of the studies objects is impossible.

\section{Feasibility of the scaling law}

Designing a terrestrial model according to the strict similarity scaling law in equation (12) ensures geometric, kinematic and dynamic similarities but fails to meet structural similarity. The system of equations always has a unique solution which means that the strict similarity scaling law is theoretically always feasible when the bound conditions (the global Mach number and the local one in the propeller tips) are met. However, the magnitude of scaling ratios in equation (12) may suggest that the resulting physical dimensions may be unrealistic. In other words, constructing a scaled model may prove to be hardly possible.

One way of assessing the feasibility of scaling is to use the so-called allometric relations. As Bejan proves in his work ${ }^{38}$ technological evolution follows physical patterns similar to its biological counterpart called constructal law. In the case of flying species, airplane evolution obeys theoretical allometric rules that strongly correlate with birds evolution. Allometric relations allow to study proportions between main characteristics such as mass, size and speed which ratios correspond to constant power laws (see Figure 2 in Bejan et al. ${ }^{38}$ ). It means that the ratio between two physical magnitudes of any airship model is the same independently of its technological level. A good example is the speed of flight which can be approximated by the Bejan's constructal theory. ${ }^{39}$ It should be correlated according to the body mass to the power $1 / 6$ as shown in equation (14). This law is expected to be accurate within one order of magnitude.

$$
\text { Speed } \sim\left(\frac{\rho_{\text {body }}}{\rho_{\text {atmosphere }}}\right)^{1 / 3} g^{1 / 2} \rho_{\text {body }}^{-1 / 6} \text { Mass }^{1 / 6}
$$

Both the designed prototype and the scaled model should follow the same law as in their design they use existing technology which should follow the trends of the state of the art. Therefore, it is possible to transform equation (14) using scaling ratios $N_{U}, N_{L}$, and $N_{M}$ giving equation (15). In this case the $\rho_{b o d y}$ is considered as Mass/Lenght ${ }^{3}$. Inserting $N_{L}$ and $N_{M}$ given in equation (12) into the RHS of equation (15) returns $N_{U}$ given in equation (12), which proves the validity of strict scaling law.

$$
N_{U} \sim \frac{N_{g}^{1 / 2}}{N_{\rho}^{1 / 3}} \frac{N_{M}^{1 / 3}}{N_{L}^{1 / 2}}
$$

The fact that every flying object (animal or manmade machines) is governed by the same power law in

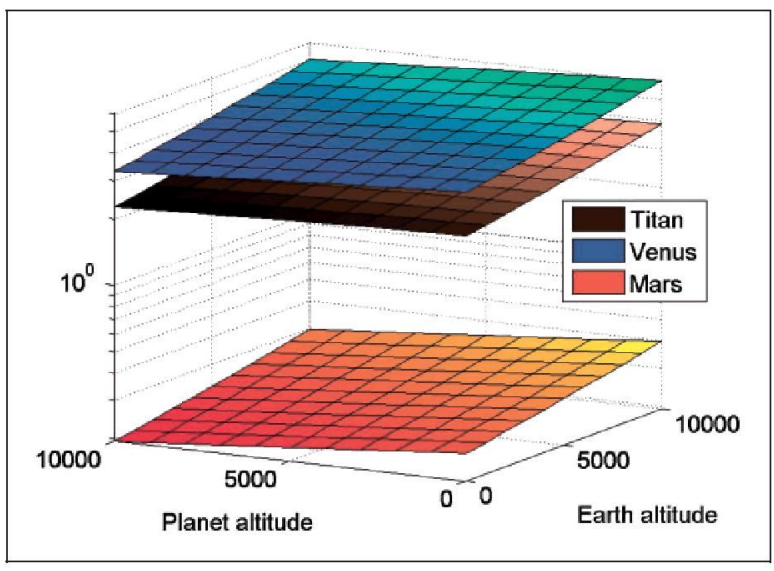

Figure I. Global Mach ratio, $N_{\text {Mach }}$. 


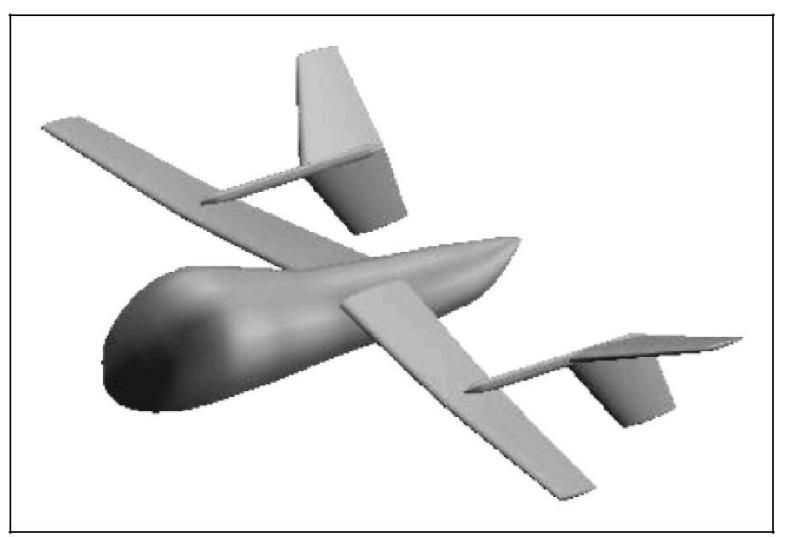

Figure 2. AVIATR prototype model.

different environments demonstrates, as Bejan states in his work, ${ }^{39}$ that the theory could be even applied to predict design evolution on other planets with different gravitational accelerations and atmospheric properties. This theory confirms again that the theoretic feasibility of the strict similarity scaling law in the incompressible case is possible, however without considering the challenge faced when constructing the scaled model. In fact, the relaxed similarity procedure should be approximated well by this theory, which will be further analyzed in "Case study" section.

It is worthwhile therefore to investigate if it can be applied to a realistic example considering the worst case scenario. Let compressibility regime be one of the criteria. It can be deduced that for the worst case scenario the speed of sound on Earth and on other object has to be the lowest and highest respectively in a given range of altitudes (in this case from 0 to $10,000 \mathrm{~m}$ ). This corresponds to the highest and lowest altitude (lowest and highest temperature) on Earth and other objects, respectively. Mars can be discarded in this case because its speed of sound is always lower than the terrestrial as shown in Figure 1. On the other hand, for Titan and Venus the required flight Mach number of the terrestrial model is higher than the object's flight Mach number, which means that in the terrestrial flight the compressibility regime can differ. Assuming Mach 0.3 to be the limiting value for incompressible flow regime, it sets the maximum speed of the terrestrial model. Using $N_{U}$ values obtained from equation (12) the maximum speed on Titan and Venus for the prototype are $16 \mathrm{~m} / \mathrm{s}$ and $21 \mathrm{~m} / \mathrm{s}$, respectively. These values represent a limit in the feasibility of the terrestrial scaled model flight tests. Using the definition of $C_{L}$ the flight speed can be defined by $U=\sqrt{\frac{W / S}{0.5 p C_{L}}}$, where $C_{L}$ and $W / S$ are specified by prototype design. Assuming a usual value of $C_{L}$ to be 0.6 and knowing that medium-size UAVs have a wing loading between 500 and $1000 \mathrm{~N} / \mathrm{m}^{2}$, the resulting flight speed range is between 19 and $27 \mathrm{~m} / \mathrm{s}$ and between 5 and $7 \mathrm{~m} / \mathrm{s}$ for Titan and Venus, respectively. In the case of Venus, the speed range is below the limit which means that any prototype with design parameters of the same order can be strictly scaled for terrestrial flight tests. However, for Titan, the velocity range exceeds the limit. This means that only prototype designs with higher $C_{L}$ or/and lower $W / S$ are feasible for terrestrial flight tests. This is a limitation because aerodynamic efficiency could coincide with lower $C_{L}$ values and higher $W / S$ is desirable for weight optimization and UAV encapsulation. Therefore, in some cases strictly scaled terrestrial models are not technologically feasible.

A careful analysis of strict similarity scaling law on a particular example will be done in "Case study" section. It is clear that the multidisciplinary strict similarity scaling law may not be feasible and scaling only certain aspects may not be the most optimal solution. As a result a different approach should be taken in order to design a scaled model which meets all the flight test restrictions in terms of accuracy of the results, their scalability and financial resources. This is where the relaxed similarity scaling law can be applied.

\section{Relaxed similarity scaling}

Relaxing the constrains defined by the strict similarity scaling law in equation (7) offers a wide range of options approximating the solution generating a map of scaling laws. The choice of relaxation variables and their ranges depends on parameter sensibility and is crucial for this optimization problem.

Firstly, the scaling function to be optimized needs to be defined. In the case of exact scaling law the problem is a system of equations with a unique solution. Adding more equations leading to new scaling conditions makes the problem redundant. In the case of defining the function for a relaxed similarity strategy, all the similarity parameters of strict similarity scaling law given in equation (7) are included in the optimization function, as well as other parameters previously neglected due to compatibility and redundancy reasons. Adding more terms, not necessarily of technical character, is possible and helps to choose the right tendency/gradient in order to select the most suitable scaling law fulfilling all the requirements.

This study proposes defining the scaling function (16) as a weighted sum of all parameters that are involved in the strict scaling law and additionally all the neglected parameters. Enforcing similarity will require certain parameters to be prioritized from the rest of the system, which is reflected in the relative weights $\left(c_{i}\right)$ between parameters. Parameter sensibility study would be required in order to choose their values.

$$
f(\mathbf{N})=\sum_{i} c_{i}\left(N_{i}-1\right)^{2}
$$

where $\mathbf{N}=\left(N_{i}\right)_{i}$

$$
i=\operatorname{Re}_{L}, F r, C_{L}, J, \operatorname{Re}_{D}, \text { Mach, } \text { Mach }_{t i p}, W / S
$$


The terms in equation (16) are given in quadratic form with the aim of finding a minimum of the scaling function. The absolute minimum occurs for all the ratios equal one representing exact similarity for the extraterrestrial and terrestrial flights. Mach number (defined for the aircraft and for the propeller) and wing loading ratio have been added to the scaling function as a compliment to the similarity parameters defined in equation (7). They introduce a restriction to the problem since similar compressibility regime should be maintained. Wing loading influences various aspects of the aerodynamic performance and has an important effect on the structure design and material selection.

Analogously to the strict similarity scaling law, the relaxed similarity scaling law is defined for a trimmed aircraft. The vertical equilibrium is once again fulfilled by setting lift equal to weight. The horizontal equilibrium requires fulfilling the condition given in equation (9). In this case $N_{R e_{L}}, N_{F r}, N_{C_{L}}, N_{R e_{D}}$, and $N_{J}$ are not necessarily equal to one, which means that $N_{C_{D}}$ and $N_{C_{T}}$ depending on those similarity parameters are also not necessarily equal to one. However, since the similarity parameters are being optimized to be one and the sensitivity to small changes of $N_{C_{D}}$ and $N_{C_{T}}$ is relatively low, it can be assumed that $N_{C_{D}} \simeq 1$ and $N_{C_{T}} \simeq 1$. As a result, they can be neglected transforming equation (9) into equation (17).

$$
\frac{N_{U}^{2} N_{L}^{2}}{N_{n}^{2} N_{D}^{4}}=N_{\text {trim }} \simeq 1
$$

Equation (17) is a condition for horizontal trim for the scaled model which needs to be fulfilled. Consequently, in order to maintain it sufficiently close to unity, it is included in the scaling function (18).

$$
\begin{aligned}
& \qquad \begin{aligned}
f(\mathbf{N})= & \sum_{i} C_{i}\left(N_{i}-1\right)^{2} \\
\text { where } \quad \mathbf{N}= & \left(N_{i}\right)_{i} \\
i= & \operatorname{Re}_{L}, F r, C_{L}, J, \operatorname{Re}_{D}, \text { Mach, Mach } \\
& W / S, \text { trim },
\end{aligned}
\end{aligned}
$$

The scaling function (18) can be further expanded by a vast range of different parameters subject to the requirements of the project, such as available financial resources and accuracy tolerances. For the purpose of this article, additional parameters will be omitted without loss of general validity of the method.

A relaxed scaling strategy allows to specify additional constrains to the optimization problem in order to find the most suitable scaling law which is a compromise between all the considerations of diverse nature. The restrictions presented in equation (19) should be considered in the optimization problem as limit bounds in the solution. Depending on the optimization method to be used, nonlinear constraints may be also included in the definition problem.

$$
N_{i, \min }<N_{i}<N_{i, \max } \quad \text { where } \quad i=U, L, M, D, n
$$

In a preliminary analysis is appeared that the weights $c_{i}$ are less important than the constrains given in equation (19). Adjusting weight values can slightly reduce the differences in model similarity, but since the bounds act on domain boundaries, selecting an area of the map of scaling laws, they influence directly the feasibility of the scaling function and its applicability. Distributing the weight values equally, so that they sum up to one, proves to be sufficient. A more detailed analysis is left for future work.

Once the weights are fixed, the nonlinear optimization problem can be addressed. The relaxed similarity scaling law is obtained from solving the nonlinear optimization problem composed of the objective function to be minimized (18) with the constrains shown in equation (19). Despite the fact that there exists a variety of optimization algorithms suitable for such a problem, a standard optimization routine based on BFGS algorithm is proposed to be applied in "Case study" section. The chosen algorithm does not affect at all the validity of the methodology and the analysis of its effects on convergence or speed is beyond the scope of this study.

\section{Case study}

This section aims to apply the scaling methodology to an example prototype and analyze different scaled models for terrestrial flight conditions. The analysis includes characterizing the difference between scaling methods by using numerical tools for the different disciplines by comparing the results to the extraterrestrial prototype.

AVIATR (mentioned in section "UAV projects") has been selected as an example prototype due to the advanced implementation degree of the project (Figure 2). The Aerial Vehicle of In-Situ and Airborne Titan Reconnaissance design is a propeller driven vehicle with classical wing-body configuration and twin tail joint to the main wing by booms. A summary of characteristics used for the example prototype modeling are shown in Table 2 .

The analysis is done for the full-size prototype in cruise conditions on Titan specified by the mission. Its corresponding scaled models are analogously analyzed in cruise conditions specified by scaling laws. Their terrestrial flight altitude is assumed to be sea level to facilitate conducting flight test. This is done despite the fact that sea level may not be optimal altitude in terms of reproducing extraterrestrial conditions. 
Firstly, the Earth's and Titan's atmosphere need to be defined. Their properties are numerically modeled as described in section "Atmosphere modeling".

To model aerodynamics, lifting surfaces are analyzed by using a Vortex Lattice Method code giving lift and induced drag. Basing on the lift distribution, 2D boundary layer viscous method integrates viscous drag along the span. The fuselage is considered as a streamlined body and, since only cruise state is analyzed, it is considered to be always horizontal to the flow and therefore does not generate lift. Its drag can be calculated using Hoerner's formula for streamlined bodies. $^{40}$

Table 2. AVIATR characteristics.

\begin{tabular}{lc}
\hline Parameters & Values \\
\hline Span $(\mathrm{m})$ & 7.13 \\
MAC $(\mathrm{m})$ & $0.5 \mathrm{I}$ \\
Fuselage length $(\mathrm{m})$ & 3.72 \\
Max. fuselage diameter $(\mathrm{m})$ & 1.04 \\
Propeller diameter $(\mathrm{m})$ & 0.48 \\
Cruise speed $(\mathrm{m} / \mathrm{s})$ & 6.23 \\
Mass $(\mathrm{kg})$ & $1 \mathrm{I} .75$ \\
Flight altitude $(\mathrm{m})$ & 8,000 \\
Atmospheric density $\left(\mathrm{kg} / \mathrm{m}^{3}\right)$ & 3.80 \\
\hline
\end{tabular}

Propeller performance is modeled with an advanced blade-element/vortex method with viscous analysis.

Structure model consists of wing loading ratio and force and moment distributions which result from aerodynamic analysis. A further step regarding structure scaling is analyzing the shear force and bending moments at the wing root. This determines whether the same structure and material can be used to construct the scaled models.

Numerical tools introduce certain errors that are neglected since only a relative comparison between the results of the prototype and its scaled models is important.

Due to scarce information about AVIATR, the wing and tails are modeled to be congruent with the original planform with no twist distribution. The profile used throughout the span is NACA 0012. To approximate the size of the prototype, the geometry was then scaled to give $C_{L}=0.6$ in cruise conditions on Titan which coincides with the point of maximum aerodynamic efficiency of the wing-tail configuration. The propeller used in the propulsion analysis was a 2-blade commercial model APC Thin Electric $19 \times 12$ of known chord, twist distribution and experimentally determined $C_{T}$ and $C_{P}$ as a function of $J .^{41}$ It was assumed to have a constant but unknown profile along the blades. The aerodynamic properties of this profile were iteratively chosen so that the propeller

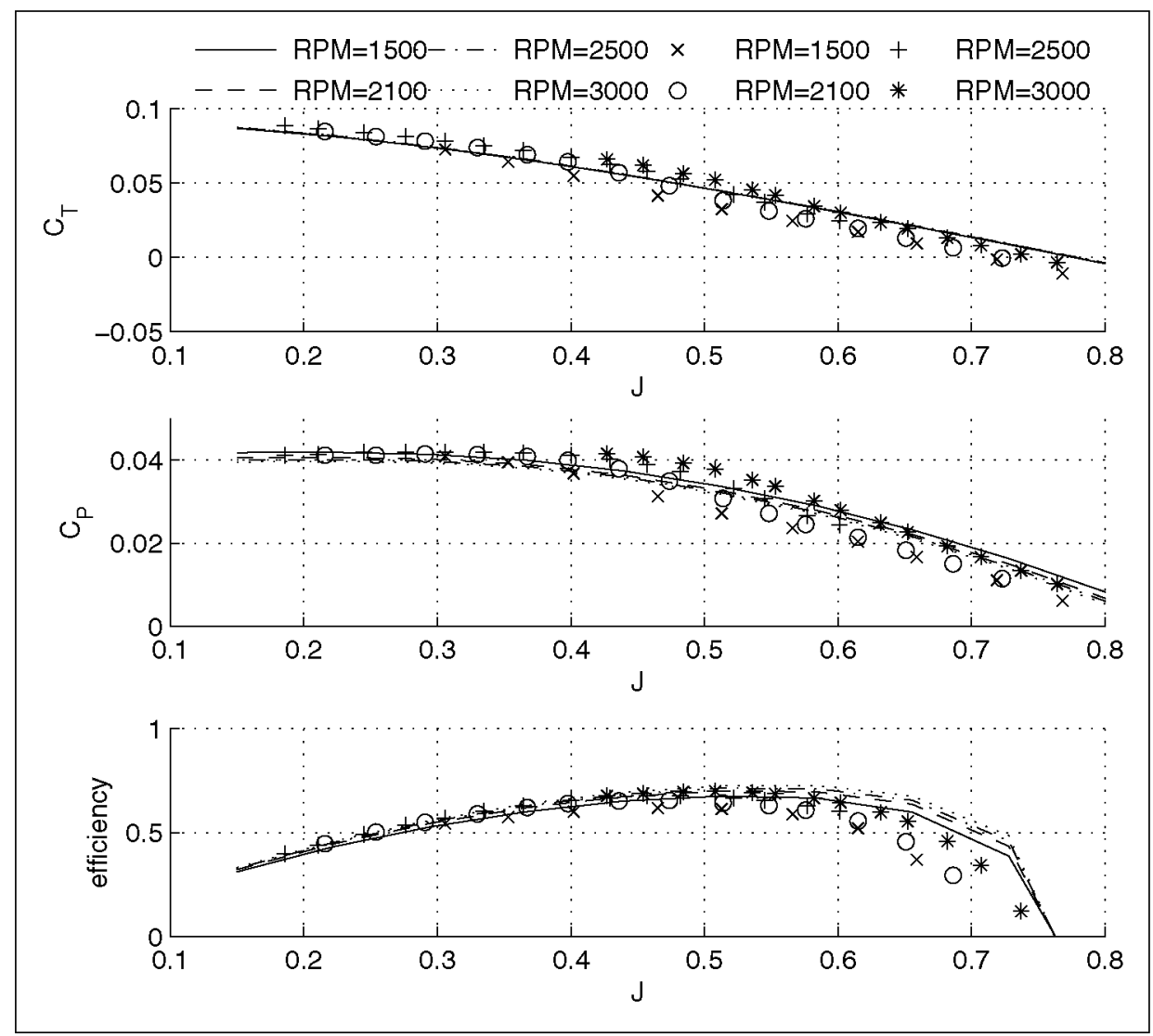

Figure 3. Propeller definition, where the lines and data points represent numerical results and experimental data, respectively. 
Table 3. Prototype in cruise condition.

\begin{tabular}{|c|c|}
\hline Parameter & Value \\
\hline \multicolumn{2}{|c|}{ Scaling variables } \\
\hline$U(\mathrm{~m} / \mathrm{s})$ & 6.2 \\
\hline$b(m)$ & 7.22 \\
\hline$m(\mathrm{~kg})$ & 115.8 \\
\hline$D(\mathrm{~m})$ & 0.48 \\
\hline $\mathrm{r} / \mathrm{min}$ & $1.8 \mathrm{e} 3$ \\
\hline \multicolumn{2}{|c|}{ Aerodynamics } \\
\hline $\operatorname{Re}_{L}$ & $2.07 e 6$ \\
\hline $\mathrm{Fr}$ & 7.49 \\
\hline$C_{L}$ & 0.592 \\
\hline Mach & 0.032 \\
\hline$C_{D}$ & 0.044 \\
\hline$C_{L} / C_{D}$ & 13.4 \\
\hline \multicolumn{2}{|l|}{ Propulsion } \\
\hline$J$ & 0.42 \\
\hline $\operatorname{Re}_{D}$ & $4.65 \mathrm{e} 6$ \\
\hline Mach $_{\text {tip }}$ & 0.238 \\
\hline$C_{T}$ & 0.059 \\
\hline$C_{P}$ & 0.033 \\
\hline$\mu_{\text {prop }}$ & 0.75 \\
\hline$T-D[N]$ & -0.2 \\
\hline \multicolumn{2}{|l|}{ Structure } \\
\hline W/S $(\mathrm{Pa})$ & 43.6 \\
\hline$F_{Z}(\mathrm{~N})$ & $0.08 \mathrm{e} 3$ \\
\hline$M_{X}(\mathrm{Nm})$ & $0.10 \mathrm{e} 3$ \\
\hline
\end{tabular}

performance matched the experimental $C_{T}$ and $C_{P}$ distributions as shown in Figure 3.

For the purpose of this article, the knowledge of the true geometry of the vehicle is not necessary as it pretends to scale any valid aircraft which is to fly in extraterrestrial atmosphere. AVIATR was chosen as a base design due to convenience reasons.

The analysis procedure begins with aerodynamic study of AVIATR on Titan (from now on referred to as prototype). The propeller is analyzed for a range of $J$ and the results are interpolated so that the thrust matches the drag. Finally, the structural parameters are calculated. The results are presented in Table 3.

The prototype is then scaled for cruise conditions at sea level on Earth using strict and relaxed similarity law described in section "Strict similarity scaling" and "Relaxed similarity scaling" respectively and its aerodynamics, propulsion and structure are analyzed. In the case of relaxed similarity scaling, a case of bounded and unbounded domain of scaling ratios is considered. The results from strict, unbounded, and bounded relaxed similarity models are shown in Tables 4-6, respectively.

Figure 4 represents graphically the aerodynamic analysis summarized in Tables 3-5. From the point of view of aerodynamics, polar and efficiency curves
Table 4. Strict model in cruise condition scaled using aerodynamics and propulsion criteria.

\begin{tabular}{|c|c|c|c|}
\hline Parameter & Value & Ratio/Differences & Value \\
\hline \multicolumn{4}{|c|}{ Scaling ratios } \\
\hline$U(\mathrm{~m} / \mathrm{s})$ & 25.5 & $N_{u}$ & 4. 10 \\
\hline$b(\mathrm{~m})$ & 16.67 & $N_{L}$ & 2.31 \\
\hline$m(\mathrm{~kg})$ & 462.5 & $N_{M}$ & 3.99 \\
\hline$D(m)$ & I.II & $N_{D}$ & 2.31 \\
\hline $\mathrm{r} / \mathrm{min}$ & $3.3 \mathrm{e} 3$ & $N_{n}$ & 1.77 \\
\hline \multicolumn{4}{|c|}{ Aerodynamics } \\
\hline $\operatorname{Re}_{L}$ & $2.07 e 6$ & $N_{\mathrm{Re}_{L}}$ & 1.00 \\
\hline $\mathrm{Fr}$ & 7.49 & $N_{F r}$ & 1.00 \\
\hline$C_{L}$ & 0.592 & $N_{C L}$ & 1.00 \\
\hline Mach & 0.075 & $N_{\text {Mach }}$ & 2.33 \\
\hline$C_{D}$ & 0.044 & $\delta c_{D}$ & $0.0 \%$ \\
\hline$C_{L} / C_{D}$ & 13.4 & $\delta C_{L} / C_{D}$ & $0.0 \%$ \\
\hline \multicolumn{4}{|l|}{ Propulsion } \\
\hline J & 0.42 & $N_{j}$ & 1.00 \\
\hline $\operatorname{Re}_{D}$ & $4.65 \mathrm{e} 6$ & $\mathrm{~N}_{\mathrm{Re}_{D}}$ & 1.00 \\
\hline Mach $_{t i p}$ & 0.556 & $N_{\text {Mach }_{i p}}$ & 2.33 \\
\hline$C_{T}$ & 0.062 & $\delta_{C T}$ & $5.4 \%$ \\
\hline$C_{P}$ & 0.035 & $\delta_{C P}$ & $6.0 \%$ \\
\hline$\mu_{\text {prop }}$ & 0.74 & $\mu_{\text {prop }}$ & $-0.6 \%$ \\
\hline$T-D(\mathrm{~N})$ & 13.5 & $\delta_{T-D}$ & $3.8 \%$ \\
\hline \multicolumn{4}{|l|}{ Structure } \\
\hline W/S (Pa) & 236.3 & $N_{W / S}$ & 5.42 \\
\hline$F_{Z}(N)$ & $2.20 \mathrm{e} 3$ & & \\
\hline$M_{X}(\mathrm{Nm})$ & $6.81 \mathrm{e} 3$ & & \\
\hline
\end{tabular}

are most representative. As can be seen in Figure 4 the curves are almost identical, meaning that the aerodynamic behavior was faithfully reproduced. The trim point for the prototype and strict model coincide, as by definition the aerodynamic behavior is exactly reproduced, but the trim point for the relaxed model moved slightly to the left due to a decrease in CL. This is a consequence of relaxing the kinematic similarity which means that the flow patterns are not exactly reproduced. This change is, however, small as $N_{C_{L}}=0.92$. It affects the trim angle, which changes from to $-0.04^{\circ}$ to $-0.52^{\circ}$ and introduces an error to $C_{D}$ and $C_{L} / C_{D}$ which equals $-2.1 \%$ and $-6.4 \%$, respectively. There is also a very significant change in $F r$ as for the relaxed model $N_{F r}=0.32$. In this case, however, $F r$ is not an important parameter. In terms of global Mach, both models are within the incompressible regime. However, the strict model has $M_{\text {tip }}=0.556$ which exceeds the incompressibility limit of 0.3 .

Figure 5 represents graphically the propulsive analysis summarized in Tables $3-5$. It is interesting to see that, although the similarity parameters have been exactly reproduced, the strict model curves do not overlap the prototype curves but approximate them well for higher $J$. This results in $5.4 \%, 6.0 \%$, and 
Table 5. Unbounded relaxed similarity scaled model.

\begin{tabular}{|c|c|c|c|}
\hline Parameter & Value & Ratio/Differences & Value \\
\hline \multicolumn{4}{|l|}{ Scaling ratios } \\
\hline$U(\mathrm{~m} / \mathrm{s})$ & 11.9 & $N_{U}$ & 1.90 \\
\hline$b(\mathrm{~m})$ & 36.88 & $N_{L}$ & 4.64 \\
\hline$m(\mathrm{~kg})$ & 369.0 & $N_{M}$ & 3.19 \\
\hline$D(\mathrm{~m})$ & 2.41 & $N_{D}$ & 5.00 \\
\hline $\mathrm{r} / \mathrm{min}$ & $0.7 e 3$ & $N_{n}$ & 0.37 \\
\hline \multicolumn{4}{|c|}{ Aerodynamics } \\
\hline $\operatorname{Re}_{L}$ & $1.93 \mathrm{e} 6$ & $N_{R_{L}}$ & 0.93 \\
\hline $\mathrm{Fr}$ & 2.46 & $N_{F r}$ & 0.32 \\
\hline$C_{L}$ & 0.543 & $N_{C L}$ & 0.92 \\
\hline Mach & 0.035 & $N_{\text {Mach }}$ & 1.08 \\
\hline$C_{D}$ & 0.043 & $\delta_{C_{D}}$ & $-2.1 \%$ \\
\hline$C_{L} / C_{D}$ & 12.5 & $\delta_{C_{L} / C_{D}}$ & $-6.4 \%$ \\
\hline \multicolumn{4}{|l|}{ Propulsion } \\
\hline J & 0.44 & $N_{j}$ & 1.04 \\
\hline $\operatorname{Re}_{D}$ & $4.48 \mathrm{e} 6$ & $N_{\mathrm{Re}_{D}}$ & 0.96 \\
\hline Mach $_{\text {tip }}$ & 0.248 & $N_{\text {Mach }{ }_{p j}}$ & 1.04 \\
\hline$C_{T}$ & 0.057 & $\delta_{C T}$ & $-4.1 \%$ \\
\hline$C_{P}$ & 0.033 & $\delta_{C P}$ & $-1.8 \%$ \\
\hline$\mu_{\text {prop }}$ & 0.76 & $\mu_{\text {prop }}$ & $1.6 \%$ \\
\hline$T-D(\mathrm{~N})$ & 9.2 & $\delta_{T-D}$ & $3.1 \%$ \\
\hline \multicolumn{4}{|l|}{ Structure } \\
\hline W/S $(\mathrm{Pa})$ & 46.7 & $N_{W / S}$ & 1.07 \\
\hline$F_{Z}(N)$ & $1.77 \mathrm{e} 3$ & & \\
\hline$M_{X}(\mathrm{Nm})$ & $1.10 \mathrm{e} 3$ & & \\
\hline
\end{tabular}

Table 6. Bounded relaxed similarity scaled model.

\begin{tabular}{|c|c|c|c|}
\hline Parameter & Value & Ratio/Differences & Value \\
\hline \multicolumn{4}{|l|}{ Scaling ratios } \\
\hline$U(\mathrm{~m} / \mathrm{s})$ & 13.9 & $N_{U}$ & 2.23 \\
\hline$b(\mathrm{~m})$ & 7.94 & $N_{L}$ & 1.10 \\
\hline$m(\mathrm{~kg})$ & 22.5 & $N_{M}$ & 0.19 \\
\hline$D(\mathrm{~m})$ & $0.7 \mid$ & $N_{D}$ & 1.46 \\
\hline $\mathrm{r} / \mathrm{min}$ & $2.5 \mathrm{e} 3$ & $N_{n}$ & 1.35 \\
\hline \multicolumn{4}{|l|}{ Aerodynamics } \\
\hline $\operatorname{Re}_{L}$ & $2.49 \mathrm{e} 6$ & $N_{\mathrm{Re}_{L}}$ & 0.26 \\
\hline $\mathrm{Fr}$ & 5.90 & $N_{\text {fr }}$ & 0.79 \\
\hline$C_{L}$ & 0.432 & $N_{C L}$ & 0.73 \\
\hline Mach & 0.041 & $N_{\text {Mach }}$ & 1.27 \\
\hline$C_{D}$ & 0.054 & $\delta_{C_{D}}$ & $21.2 \%$ \\
\hline$C_{L} / C_{D}$ & 8.1 & $\delta_{C_{L} / C_{D}}$ & $-39.7 \%$ \\
\hline \multicolumn{4}{|l|}{ Propulsion } \\
\hline$J$ & 0.47 & $N_{j}$ & 1.13 \\
\hline $\operatorname{Re}_{D}$ & $1.42 \mathrm{e} 6$ & $N_{R_{D}}$ & 0.30 \\
\hline $\mathrm{Mach}_{\text {tip }}$ & 0.269 & $N_{\text {Mach }_{i p p}}$ & 1.13 \\
\hline$C_{T}$ & 0.051 & $\delta_{C_{T}}$ & $-13.4 \%$ \\
\hline$C_{P}$ & 0.033 & $\delta_{C_{p}}$ & $-1.5 \%$ \\
\hline$\mu_{\text {prop }}$ & 0.74 & $\mu_{\text {prop }}$ & $-1.0 \%$ \\
\hline$T-D(N)$ & -0.5 & $\delta_{T-D}$ & $-2.0 \%$ \\
\hline \multicolumn{4}{|l|}{ Structure } \\
\hline W/S $(\mathrm{Pa})$ & 50.9 & $N_{W / S}$ & 1.17 \\
\hline$F_{Z}(\mathrm{~N})$ & $0.1 \mid e 3$ & & \\
\hline$M_{X}(\mathrm{Nm})$ & $0.17 e 3$ & & \\
\hline
\end{tabular}

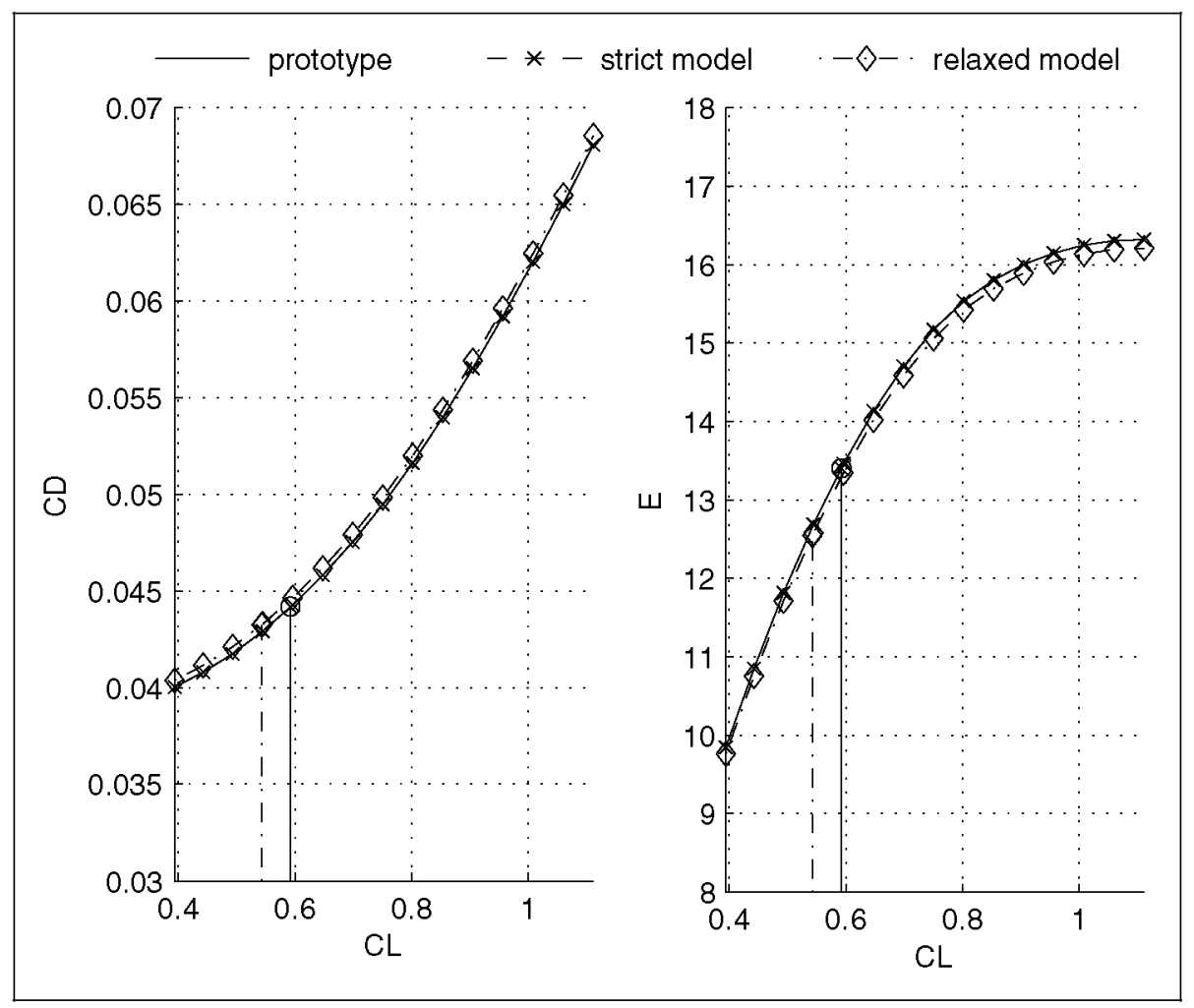

Figure 4. Aerodynamics analysis results, the vertical lines represent trim condition. 


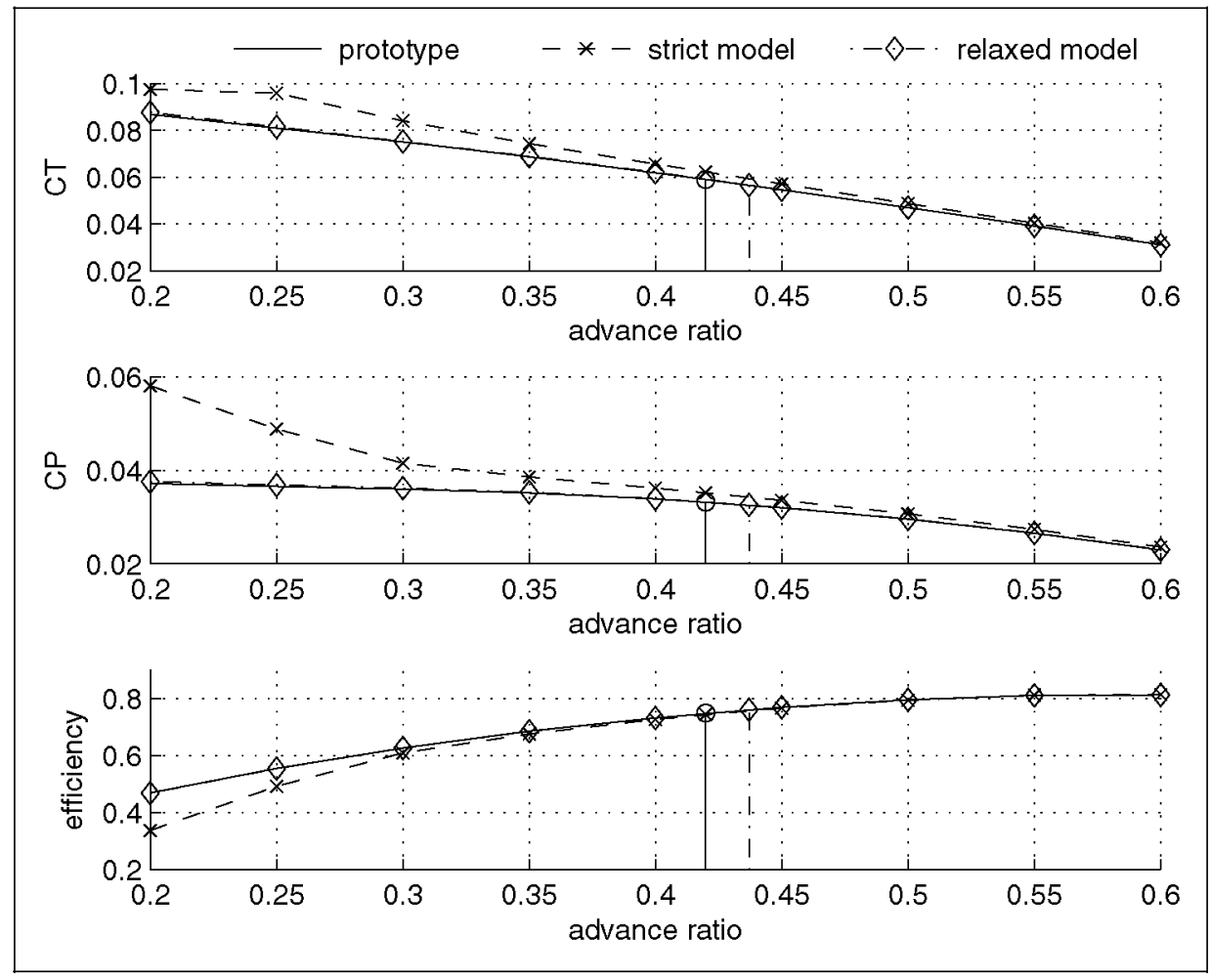

Figure 5. Propeller analysis results, the vertical lines represent trim condition.

$-0.6 \%$ error for $C_{T}, C_{P}$, and $\mu_{\text {prop }}$ respectively and $13.5 \mathrm{~N}$ surplus in thrust. This may be caused by a significant change in inflow and rotational speed. On the other hand, the relaxed model curves overlap the prototype curves but the trim point shifts slightly to the right. In this case, the similarity parameters were well reproduced as $N_{J}$ and $N_{\operatorname{Re}_{D}}$ equal 1.04 and 0.96 respectively which results in $-4.1 \%,-1.8 \%$, and $3.1 \%$ error in $C_{T}, C_{P}$, and $\mu_{\text {prop }}$, respectively.

Regarding structural analysis, it is clear that the strict model does not reliably reproduce the same conditions as the wing loading is more than five times greater. For both models the shear force $F_{Z}$ at the wing root is of the same order but the shear stresses $\sigma_{Z}$ depend on the cross-sectional surface which, assuming that the design of structural components remains the same and is only scaled, is proportional to $N_{L}^{2}$. Defining shear stress ratio as $N_{\sigma_{Z}}=\frac{\left.F_{Z}\right|_{\text {model }}}{\left.F_{Z}\right|_{\text {prototype }} N_{L}^{2}}$ gives 5.42 and 1.08 for the strict and relaxed model respectively. Analogously, for bending moment $M_{X}$ at the wing root being proportional to $N_{L}^{3}$, tensile $/ \mathrm{com}$ pressive stress ratio can be defined as $N_{\sigma}=\frac{\left.M_{X}\right|_{\text {model }}}{\left.M_{X}\right|_{\text {prototype }} N_{L}^{3}}$ giving 5.42 and 1.08 for the strict and relaxed model, respectively. Those values correspond to $N_{W / S}$ and mean that if the material used for the structural components is at its structural limit, the strict model would need to be constructed from a different material or the design of the structural components would need to be changed.
Analyzing the construction feasibility and its cost, the strict and relaxed model has a mass of 462.5 and $369.0 \mathrm{~kg}$ respectively which is correspondingly 4.0 and 3.2 times more than the prototype. Additionally, both have much higher span and in the case of the relaxed model, is it 4.6 time greater giving $36.9 \mathrm{~m}$, comparable to the size of a commercial aircraft such as the A $320 .{ }^{42}$ Although constructing such an aircraft is possible, it would probably cost more that the prototype itself. This, depending on the project's budget, may be not possible.

Fortunately, relaxed similarity scaling law allows introducing restrictions. Having previously commented, that the main possible problem of the scaled model was its size, it was decided to bound $N_{L}$ between 0.9 and 1.1 .

From Table 6 it is clear that bounding $N_{L}$ increases the similarity reproduction difference. Firstly, the aerodynamics is severely affected as $N_{R e_{L}}=0.26$ and $N_{C_{L}}=0.73$ which influence the dynamic and kinematic similarity resulting in $21.2 \%$ and $-39.7 \%$ difference in $C_{D}$ and $C_{L} / C_{D}$, respectively. Regarding propulsive similarity, $N_{R e_{D}}$ has been significantly impacted as its magnitude decreased to 0.30 . Since the remaining similarity parameters have not been greatly affected, the differences in $C_{T}, C_{P}$, and $\mu_{\text {prop }}$, equal to $-13.4 \%,-1.5 \%$, and $-1.0 \%$ respectively, are relatively low. The thrust deficit is also minimal. Keeping in mind the very restrictive $N_{L}, N_{W / S}=1.17$ means that the structural response is almost unaffected. 
Finally, is interesting to check if equation (15) is also satisfied by the relaxed scaled models. By inserting values from Tables 5 and 6 into the RHS of equation (15), the value of $N_{U}$ is 1.2 and 1.0 respectively, whereas the true values are 1.9 for unbounded and 2.2 for bounded. Both solutions maintain the same order of magnitude. Nevertheless, equation (15) can be included in the optimization problem as a nonlinear constraint.

\section{Conclusion}

Strict similarity scaling law has been shown to be incapable of reproducing simultaneously the behavior of all the analyzed disciplines. It has also been shown that it can fail in maintaining the same compressibility regime. Relaxed similarity scaling law overcomes these problems but introduces differences to reproduction of the general behavior of the plane. However, it is possible to control those differences by introducing additional terms to the scaling function and by appropriately choosing their weights which forms part of future developments. In the case, when the relaxed scaling model is infeasible to construct, it is possible to introduce ratio bounds and constraints which, although introduce more differences, allow to tailor the scaled model design to the project's needs.

\section{Conflict of interest}

None declared.

\section{Funding}

This research received no specific grant from any funding agency in the public, commercial, or not-for-profit sectors.

\section{References}

1. Savu G. Earth-mars similarity criteria for exploring martian vehicles. Acta Astronaut 2006; 59: 734-741.

2. Liu T. Comparative scaling of flapping-and fixed-wing flyers. AIAA $J$ 2006; 44: 24-33.

3. Lorenz RD. Flight power scaling of airplanes, airships, and helicopters: Application to planetary exploration. $J$ Aircraft 2001; 38: 208-214.

4. Yarin LP. The Pi-theorem, experimental fluid mechanics. Xxvi ed. Berlin: Springer-Verlag, 2012.

5. Mitcheltree RA. Dynamic scaling for earth based testing of mars terminal descent dynamics. In: $A I A A$ Atmospheric flight mechanics conference, Austin, TX, USA, 11-14 August 2003.

6. Williams DA. NASA's planetary aeolian laboratory: Exploring aeolian processes on earth, mars, and titan. Woodlands, TX, 2013.

7. Ledé JC, Croom MA and Parks RW. High altitude drop testing in mars relevant conditions for the ares mars scout mission. In: 2nd AIAA "Unmanned Unlimited" conference and workshop \& exhibit, San Diego, CA, USA, 2003.

8. Steltzner A, Bruno R, Mitcheltree R, et al. Opportunities and limitations in low-earth subsonic testing and qualification of extraterrestrial supersonic parachute designs. In: Al7th AIAA Aerodynamic decelerator systems technology conference and seminar, Monterey, CA, USA, 2003, pp.10.2514/6.2003-2135.
9. Liu T, Oyama A and Fujii K. Scaling analysis of propeller-driven aircraft for mars exploration. $J$ Aircraft 2013; 50: 1593-1604.

10. Barnes JW, McKay C, Lemke L, et al. AVIATR: Aerial vehicle for in-situ and airborne titan reconnaissance. In: 4lst Lunar and planetary science conference, The Woodlands, TX, USA, 2010.

11. Barnes JW, Lemke L, Foch R, et al. AVIATR aerial vehicle for in-situ and airborne titan reconnaissance, a titan airplane mission concept. Exp Astronomy 2012; 33: 55-127.

12. Sagdeev RZ, Linkin VM, Blamont JE, et al. The vega venus balloon experiment. Science 1986; 231: 1407-1408.

13. Lebreton JP and Matson DL. The Huygens probe: Science, payload and mission overview. Space Sci Rev 2002; 104: 59-100.

14. Pankine AA, Aaron KM, Heun MK, et al. Exploring planets with directed aerial robot explorers. AIP Conf Proc 2004; 699: 883-892.

15. Lorenz RD. A review of balloon concepts for titan. $J \mathrm{Br}$ Interplan Soc 2008; 61: 2-13.

16. Lorenz RD. Post-Cassini exploration of titan: Science rationale and mission concepts. $J$ Br Interplan Soc 2000; 53: $218-234$.

17. Dorrington GE. Concept options for the aerial survey of titan. Adv Space Res 2011; 47: 1-19.

18. Gasbarre JF, Wright $\mathrm{H}$ and Lewis MJ. A design comparison of atmospheric flight vehicles for the exploration of titan. In: AIAA Atmospheric flight mechanics conference, San Francisco, CA, USA, 15-18 August 2005.

19. Colozza A. Evaluation of an airship for planetary atmospheric exploration. In: $3 r d$ AIAA "Unmanned Unimited" technical conference, workshop \& exhibit, Chicago, IL, USA, 2004.

20. Rhew RD, Guynn MD, Yetter JA, et al. Planetary flight vehicles (PFV): Technology development plans for new robotic explorers. In: $A I A A$ Infotech@Aerospace Conferences, Arlington, VA, USA, September 2005.

21. Young LA and Aiken EW. Exploration: Past and future contributions of the vertical lift community and the flight vehicle research and technology division. In: $I^{\text {st }}$ Space exploration conference: Continuing the Voyage of Discovery, Orlando, FL, USA, 30 January to 1 February 2005.

22. Miralles C and Maccready PB. Mission concepts utilizing gliders for atmospheric and surface science. In: $30 t h$ Annual lunar and planetary science conference, Houston, TX, USA, 15-29 March 1999.

23. Levine JS, Blaney DL, Connerney JE, et al. Science from a mars airplane: The aerial regional-scale environmental survey (ARES) of mars. In: $2^{\text {nd }}$ AIAA "Unmanned Unlimited" systems, technologies, and operations. San Diego, CA, USA, 15-18 September 2003.

24. Levine JS, Croom MA, Wright HS, et al. The aerial regional-scale environmental surveyor (ARES): New mars science to reduce human risk and prepare for the human exploration. In: Concepts and approaches for mars exploration, Houston, TX, USA, 12-14 June 2012.

25. Lorenz RD. Titan bumblebee: A $1 \mathrm{~kg}$ lander-launched UAV concept. $J$ Br Interplanet Soc 2008; 61: 118-124.

26. Lafleur JM. Derivation and application of a method for first-order estimation of planetary aerial vehicle power 
requirements. In: 4th Interplanetary probe workshop, Pasadena, CA, USA, 2006.

27. Lemke L. A $100-\mathrm{kg}$ class titan airplane mission. In: 6 th International planetary probe workshop conference, Atlanta, GA, USA, 2008.

28. Wilson CF, Chassefière E, Hinglais E, et al. The 2010 European Venus Explorer (EVE) mission proposal. Exp Astronomy 2012; 33: 305-335.

29. Landis GA, Colozza A and Lamarre CM. Atmospheric flight on venus: A conceptual design. $J$ Spacecraft Rockets 2003; 40: 672-677.

30. Models of mars' atmosphere. In: NASA Space Vehicle Design Criteria (Environment), NASA SP-8010, 1974.

31. Albee AL, Arvidson R, Palluconi F, et al. Overview of the mars global surveyor mission. $J$ Geophys Res 2001; 106: 23291-23316.

32. www.grc.nasa.gov. In NASA Official WebSite.

33. Fulchignoni M, Ferri F, Angrilli F, et al. In situ measurements of the physical characteristics of titan's environment. Nature 2005; 438: 785-791.

34. Lellouch E and Hunten DM. The Lellouch-Hunten models for titan's atmosphere. In: Huygens: Science, Payload and Mission, Proceedings of an ESA conference, 1997, PP. 237-242.

35. Avduevsky VS, Ya Marov M and Rozhdestvensky MK. A tentative model of the venus atmosphere based on the measurements of veneras 5 and 6. J Atmos Sci 1970; 27: 561-568.

36. Sutherland W. The viscosity of gases and molecular force. Philos Mag Ser 1893; 36: 507-531.

37. Buckingham E. On physically similar systems: Illustrations of the use of dimensional equations. Phys Rev 1914; 4(2): 345-376.

38. Bejan A, Charles JD and Lorente S. The evolution of airplanes. $J$ Appl Phys 2014; 116: 1-6.

39. Bejan A and Marden JH. Unifying constructal theory for scale effects in running, swimming and flying. $J$ Exp Biol 2006; 209: 238-248.

40. Hoerner SF. Fluid-Dynamic drag. 1965 ed. New York: Hoerner Fluid Dynamics, 1965.

41. Brandt JB and Selig MS. Propeller performance data at low Reynolds numbers. In: $49^{\text {th }}$ AIAA aerospace sciences meeting. Orlando, FL, USA, 2011.

42. Jackson P. Jane's: All the World's Aircraft 2008-2009. 99th ed. Coulsdon, UK: Jane's Information Group Limited, 2008.

\section{Appendix}

\section{Notation}

$a$

$c_{i}$

$g$

$n$

$C_{D}$

$C_{F_{a}}$

$C_{L}$

$C_{T}$

D

$F_{a}$

$F r$

$F_{Z}$

J

K

L

$M$

Mach

Mach $_{\text {tip }}$

$M_{X}$

$N_{i}$

$R e_{D}$

$R e_{L}$

$S_{\text {ref }}$

$T$

$T-D$

$U$

$W / S$

$\alpha$

$\mu$

$\mu_{\text {prop }}$

$v$

$\rho$

$\sigma_{Z}$ speed of sound $(\mathrm{m} / \mathrm{s})$

relaxed scaling weight coefficient

gravitational acceleration $(\mathrm{m} / \mathrm{s})$

propeller rotational speed $(\mathrm{r} / \mathrm{s})$

drag coefficient

coefficient of aerodynamic force

lift coefficient

coefficient of thrust

propeller diameter $(\mathrm{m})$

resultant aerodynamic force $(\mathrm{N})$

Froude number

shear force at the wing $\operatorname{root}(\mathrm{N})$

advance ratio

fluid bulk elasticity modulus $\left(\mathrm{N} / \mathrm{m}^{2}\right)$

characteristic length (m)

model mass $(\mathrm{kg})$

Mach number

Mach number at the propeller tip

bending moment at the wing root $(\mathrm{Nm})$

ratio of parameter $i$ between the Earth

and a given celestial body

Reynolds number based on propeller

diameter

Reynolds number based on character-

istic length $L$

reference area $\left(\mathrm{m}^{2}\right)$

thrust $(\mathrm{N})$

thrust deficit $(\mathrm{N})$

freestream velocity $(\mathrm{m} / \mathrm{s})$

wing loading $\left(\mathrm{N} / \mathrm{m}^{2}\right)$

angle of attack (rad)

atmospheric dynamic viscosity $(\mathrm{kg} / \mathrm{s} / \mathrm{m})$

propulsive efficiency

atmospheric kinematic viscosity $\left(\mathrm{m}^{2} / \mathrm{s}\right)$

atmospheric density $\left(\mathrm{kg} / \mathrm{m}^{3}\right)$

shear stress at the wing root $\left(\mathrm{N} / \mathrm{m}^{2}\right)$ 